\title{
Inferring the origins of the pulsed $\gamma$-ray emission from the Crab pulsar with ten-year Fermi-LAT data (Corrigendum)
}

\author{
Paul K. H. Yeung ${ }^{1,2}$ \\ ${ }^{1}$ Institute of Experimental Physics, Department of Physics, University of Hamburg, Luruper Chaussee 149, 22761 Hamburg, \\ Germany \\ e-mail: kin.hang.yeung@desy.de \\ 2 Nicolaus Copernicus Astronomical Center, Polish Academy of Sciences, Rabiańska 8, 87-100 Toruń, Poland
}

A\&A 640, A43 (2020), https://doi.org/10.1051/0004-6361/202038166

Key words. pulsars: individual: Crab pulsar - gamma rays: stars - errata, addenda

This is a corrigendum to the original article Yeung (2020) and is mostly adapted from Appendix C.4 of the author's Ph.D. Thesis (Yeung 2021).

In Sect. 3.4 of the original article, we deduced from the power law with a scaling factor (PLSF) fitting result that $\Gamma$ for $P 2_{M}$ is lower than that for $P 1_{M}$. Nevertheless, the previous calculation of the significance of this difference did not consider the covariance of the $\Gamma$ values for $P 1_{M}$ and $P 2_{M}$ (it should be noted that the spectral data for $P 1_{M}$ and $P 2_{M}$ are fit simultaneously such that their solutions share the same scaling factor $\epsilon$ ). After taking this covariance into account, which is computed to be 0.010 , we now determine that $\Gamma$ for $P 2_{M}$ is lower than that for $P 1_{M}$ by $0.35 \pm 0.08$, which corresponds to $\sim 4.2 \sigma$ (much higher than the $\sim 2.1 \sigma$ reported in the original paper). Additionally, Fig. 1 demonstrates that the $4 \sigma$ confidence region of $\Gamma$ values for $P 1_{M}$ and $P 2_{M}$ excludes all equal-value combinations.

The revised significance strengthens the argument that the flux ratio of $P 1$ to $P 2$ still decreases with photon energy even above $10 \mathrm{GeV}$. This, in turn, strengthens the interpretation that there are anisotropic pulsar winds that could lead to the observed harder spectrum at $P 2_{M}$ relative to $P 1_{M}$ (as put forward by Aharonian et al. 2012).

Moreover, taking the statistical errors into account, the $\epsilon$ values obtained for MAGIC and VERITAS (both 1.22) are basically consistent with the estimated systematic errors of around $15 \%$ on energy scales of IACT telescopes under excellent atmospheric conditions (e.g., Aharonian et al. 2006; Aleksić et al. 2016). This provides further confirmation for the physical reasonableness of those fitting results.

\section{References}

Aharonian, F., Akhperjanian, A. G., Bazer-Bachi, A. R., et al. 2006, A\&A, 457, 899

Aharonian, F. A., Bogovalov, S. V., \& Khangulyan, D. 2012, Nature, 482, 507

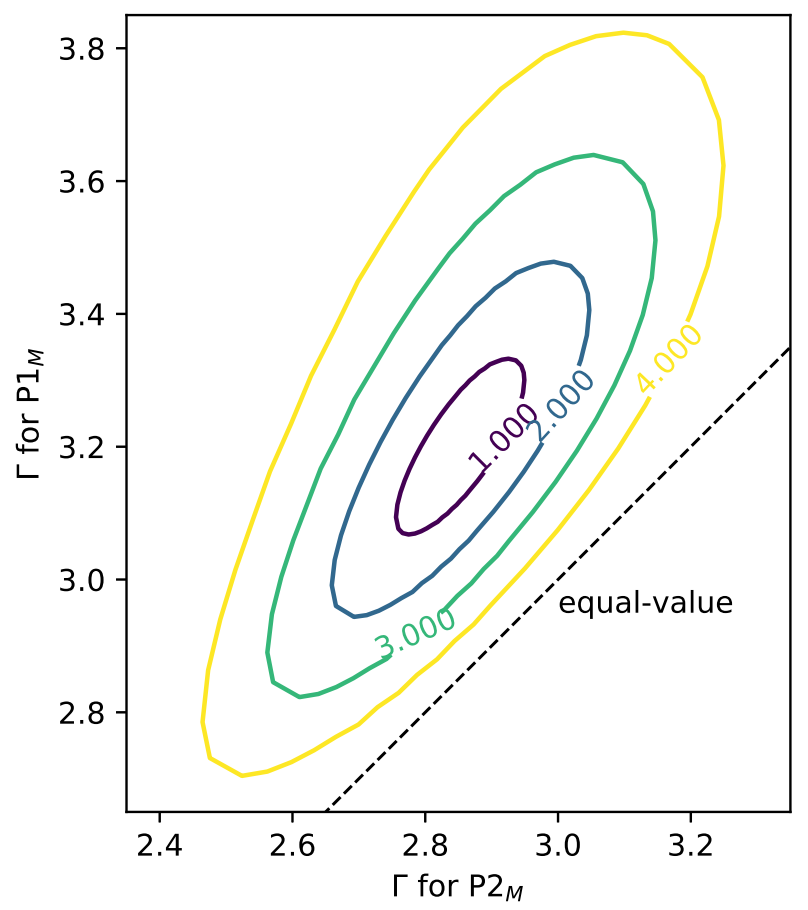

Fig. 1. Confidence regions of $\Gamma$ values for $P 1_{M}$ and $P 2_{M}$. The plotted covariance contours respectively correspond to the significance levels of $1 \sigma, 2 \sigma, 3 \sigma$, and $4 \sigma$ (from innermost to outermost). The reference line (which is black, dashed, and straight) indicates the combinations of equal $\Gamma$ values.

Aleksić, J., Ansoldi, S., Antonelli, L. A., et al. 2016, Astroparticle Physics, 72, 76

Yeung, K. H. 2021, PhD thesis, Staats-und Universitätsbibliothek Hamburg Carl von Ossietzky

Yeung, P. K. H. 2020, A\&A, 640, A43 